\title{
Effects of mineralocorticoid receptor antagonists in patients with preserved ejection fraction: a meta-analysis of randomized clinical trials
}

Yanmei Chen, He Wang, Yongkang Lu, Xiaobo Huang, Yulin Liao and Jianping Bin*

\begin{abstract}
Background: Mineralocorticoid receptor antagonists (MRAs) have been shown to be effective in patients with heart failure or myocardial infarction complicated by a reduced ejection fraction. However, the role of MRAs in patients with preserved ejection fraction (PEF) remains to be clarified. We aimed to summarize the evidence for the efficacy of MRAs in patients with either heart failure with PEF (HF-PEF) or myocardial infarction with PEF (MI-PEF).
\end{abstract}

Methods: We searched PubMed, EMBASE, Cochrane Library, and clinical trials databases for randomized controlled trials, through June 2014, assessing MRA treatment in HF-PEF or MI-PEF patients. Fourteen randomized controlled trials (MI-PEF, 5; HF-PEF, 9; $\mathrm{n}=6,428$ patients) were included.

Results: MRA treatment reduced the risk of hospitalization for heart failure (relative risk, $0.83 ; 95 \%$ confidence interval [Cl], 0.70 to 0.98 ), improved quality of life (weighted mean difference [WMD], $-5.16 ; 95 \% \mathrm{Cl},-8.03$ to -2.30 ), left ventricular end-diastolic diameter (standardized mean difference, $-0.21 ; 95 \% \mathrm{Cl}, 0.32$ to -0.11 ), and serum amino-terminal peptide of procollagen type-III level (WMD, $-1.50,95 \% \mathrm{Cl},-1.72$ to -1.29$)$ in patients with PEF. In addition, MRAs reduced E/e'(an echocardiographic estimate of filling pressure for assessment of diastolic function; WMD,$-1.82 ; 95 \% \mathrm{Cl},-2.23$ to -1.42 ) in HF-PEF patients and E/A ratio (the ratio of early to late diastolic transmitral flow; WMD, $0.12 ; 95 \% \mathrm{Cl}, 0.10$ to 0.14$)$ in MI-PEF patients. However, all-cause mortality was not improved by MRAs in either HF-PEF $(P=0.90)$ or MI-PEF $(P=0.27)$ patients.

Conclusions: MRA treatment in PEF patients led to reduced hospitalization for heart failure, quantifiable improvements in quality of life and diastolic function, and reversal of cardiac remodeling, but did not provide any all-cause mortality benefit.

Keywords: Meta-analysis, Mineralocorticoid receptor antagonists, Preserved ejection fraction, Randomized controlled trial

\section{Background}

Approximately half of patients with heart failure (HF) have normal or only mildly impaired left ventricular ejection fractions (LVEFs) [1,2]. Patients with this profile, known as HF with preserved ejection fraction (HF-PEF), have signs, symptoms, quality of life (QoL), and prognoses similar to HF patients with a reduced ejection fraction (HF-REF) [3,4]. Furthermore, patients with acute myocardial infarction (MI) often have preserved ejection fraction

\footnotetext{
*Correspondence: jianpingbin@126.com

State Key Lab for Organ Failure Research, Department of Cardiology, Nanfang Hospital, Southern Medical University, 1838 North Guangzhou Avenue, Guangzhou 510515, China
}

\section{Biomed Central}

(PEF) [5]. Although many medical therapies benefit HF patients and post-MI patients with reduced LVEF [6], effective, evidence-based pharmacologic treatments are not currently available for PEF patients [7].

Aldeosterone-based activation of mineralocorticoid receptors has been demonstrated to contribute to the pathogenesis of HF and adverse cardiac remodeling after MI through multiple mechanisms, mainly including sympathetic activation, promotion of cardiac and vascular fibrosis, endothelial dysfunction, sodium retention, and potassium loss $[8,9]$. Mineralocorticoid receptor antagonists (MRAs) may inhibit these deleterious effects [10] 
and may contribute to a beneficial therapeutic strategy for PEF patients. MRAs are effective for reducing total and cardiovascular mortality in patients with HF-REF (LVEF $<35 \%)$ and post-MI patients with left ventricular dysfunction (LVEF <40\%) [11-13]. However, whether they have a role in PEF remains to be clarified.

A recent series of studies assessed the efficacy of MRAs in HF-PEF patients and in patients with PEF after MI (MI-PEF) [14-19]. Although some studies failed to show a significant mortality benefit for MRA use [14,15], a number demonstrated a range of secondary benefits such as improved QoL, diastolic function, and cardiac remodeling, in response to MRA therapy [16-19]. As patients with PEF are usually older than HF-REF patients, a comprehensive evaluation may help provide support for therapy that improves symptoms and QoL, rather than mortality. In addition, since diastolic dysfunction and cardiac remodeling are considered the major underlying cardiac pathophysiology in HF-PEF and MI-PEF [20], combining data regarding the impact of MRAs on these related parameters might elucidate some encouraging findings. However, data combining the experience from published randomized controlled trials to evaluate the effects of MRAs in PEF patients do not exist. Given the limited evidence concerning MRAs in PEF patients, this meta-analysis aimed to summarize the available data from randomized controlled trials (RCTs) to determine the efficacy and safety of MRAs in PEF (including both HF-PEF and MI-PEF) patients.

\section{Methods}

This meta-analysis was performed and reported according to the Preferred Reporting Items for Systematic Reviews and Meta-Analyses (PRISMA) guidelines (Additional file 1) [21].

\section{Literature search}

We searched the MEDLINE, EMBASE, Cochrane Library databases, and clinical trials databases (clinicaltrials.gov, controlled-trials.com, and clinicaltrialsregister.eu) for randomized controlled trials conducted between January 2000 and June 2014, using the following key words: i) mineralocorticoid receptor antagonists, aldosterone receptor antagonist, canrenoate, canrenoate potassium, canrenone, canrenoic acid, spironolactone, or eplerenone; ii) preserved left ventricular function, preserved ejection fraction, heart failure with normal ejection fraction, or diastolic heart failure; and iii) randomized controlled trials. Our literature search was limited to studies involving human subjects, reported in English. The list of full search strategies for EMBASE and MEDLINE is provided in Additional file 2. The search strategies for other databases are available on request.

\section{Inclusion criteria}

We included prospective, RCTs that: i) enrolled adult PEF patients with LVEFs $\geq 40 \%$ (including post-MI patients and those with symptomatic or asymptomatic HF), ii) assigned patients to MRA treatment versus placebo or control, iii) had at least one of the clinical outcomes of interest, and iv) had a study duration of at least 4 months.

\section{Data extraction}

Two independent reviewers screened all titles and the abstracts of all citations; potentially relevant articles were assessed according to the inclusion criteria. Disagreements were discussed until a consensus on inclusion/exclusion was reached. Information on patient characteristics, study design, quality, intervention strategies, and clinical outcomes was systematically extracted from each report using a standardized form. Data regarding safety and adverse events, including hyperkalemia and gynecomastia, were also noted. Hyperkalemia was defined as a potassium level $>5.5 \mathrm{mmol} / \mathrm{L}$. We used definitions of renal failure and gynecomastia as per the primary trial publication. The quality of the included RCTs was assessed using the Jadad quality scale [22].

\section{Outcome measures}

The clinical outcomes for this meta-analysis were allcause mortality and hospitalization due to HF. We also assessed echocardiographic parameters related to diastolic function, including E/e' (an estimate of filling pressure used to assess diastolic function), E/A (the ratio of early to late diastolic transmitral flow), E-wave deceleration time, and isovolumic relaxation time (IVRT) andvariables related to left ventricular structure and function, including LVEF, left ventricular end-diastolic volume index, left atrial volume index (LAVI), and left ventricular mass index (LVMI). More importantly, we also assessed relevant outcomes in terms of serum indicators and functional capacities: B-type natriuretic peptide, amino-terminal peptide of procollagen type-III (PIIINP), QoL, and 6-min walking distance.

\section{Statistical analysis}

For categorical variables, we calculated the relative risk (RR) and the absolute risk reduction (RD), as well as the corresponding 95\% confidence intervals (CIs) for the outcome variables of interest, using the DerSimonian and Laird random effects model. Quantitative outcomes changing between baseline and follow-up were summarized and compared between the treatment and control groups using the weighted mean difference (WMD) and 95\% CI, unless the outcomes used different scales, when the standardized mean difference (SMD) and 95\% CI were used. The random-effects model using the DerSimonian and 
Laird method was used irrespective of heterogeneity because we anticipated heterogeneity between the trials [23]. A priori, we defined significant heterogeneity between trials as an $\mathrm{I}^{2}$ value of $>50 \%$ [24]. We assessed the evidence of publication bias using a funnel plot with an Eggers test [25]. A two-sided $P$ value $<0.05$ was considered statistically significant for all analyses.

Predefined subgroup analyses were conducted, a priori, according to the PEF subtypes (HF-PEF and MI-PEF), treatment durations ( 6 to 11 months, and $\geq 12$ months), and MRA agent used (spironolactone, canrenoate, oreplerenone). If a given trial could be split into two or more separate studies, based on different treatment time points, the study with the longest follow-up was included in the meta-analyses. If a given trial could be split into two MRA groups with different doses, the group receiving the standard dose was included in the meta-analyses. Sensitivity analyses were conducted using sequential omission of a single study from the total studies and evaluating the influence of each study on the pooled effect estimates. All analyses were performed using Stata, version 11.2 (Stata Corp, College Station, TX, USA).

\section{Results}

Figure 1 displays the study selection flow diagram. The primary search for randomized clinical trials on MRAs and PEF generated 1,422 potentially relevant articles. After screening the titles and abstracts of all studies, 121 full-text articles met the general inclusion criteria and were reviewed for strict inclusion or exclusion criteria. Finally, 14 RCTs were included [5,14-19,26-32].

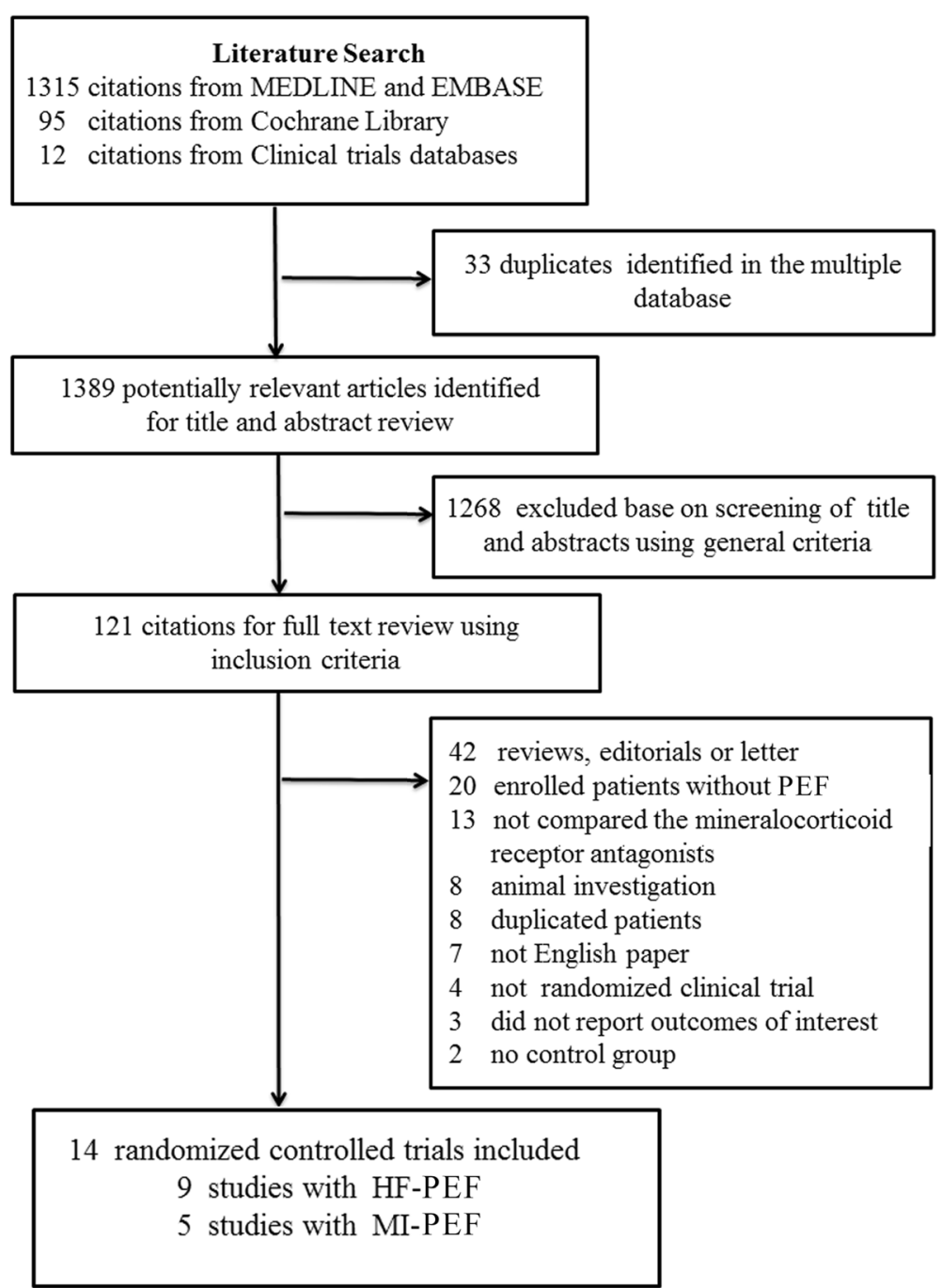

Figure 1 Flowchart of study search and selection in this meta-analysis. MI, Myocardial infarction; MRAs, Mineralocorticoid receptor antagonists; RCTs, Randomized controlled trials. 


\section{Study characteristics and quality}

All trials (Table 1) were parallel arm trials, with a mean ( \pm SD) duration of $11.75 \pm 10.36$ (range, 6 to39.6) months. Of the included trials, nine were conducted in HF-PEF $(\mathrm{n}=4,127)[14,16,26-32]$ and five $(\mathrm{n}=2,301)$ in MI-PEF patients $[5,15,17-19]$. Thus, a total of 6,428 participants were randomly assigned to receive MRAs $(n=3,249)$, placebo $(n=2,861)$, standard therapy $(n=301)$, or active comparator treatment $(\mathrm{n}=17)$. The withdrawal rates were comparable between the MRA treatment groups (9.86\%) and control groups $(10.79 \%)$. Spironolactone was the predominant MRA used (eight trials) [5,14,16,19,27-29,32], followed by eplerenone (four trials) $[15,17,30,31]$ and canrenoate (two trials) $[18,26]$. Placebo controls were used in all except four trials. Of these four trials, one used active comparators (ACE inhibitor [26]) and the other three used standard therapy $[5,17,19]$. Background medical therapy was inconsistently reported. The Treatment of Preserved Cardiac Function Heart Failure with an Aldosterone Antagonist (TOPCAT) trial [14] accounted for more than half of the patients in this meta-analysis. The Jadad scores of individual trials ranged from 1 to 5 (mean, $3.36 \pm 1.34$ ) and only three trials $[16,17,32]$ had clear allocation concealment (Additional file 3: Table S1). Patient characteristics for the included studies are presented in Additional file 3: Tables S2 and S3.

\section{Effect of MRAs on clinical outcomes}

The combined data from the 14 RCTs did not show a significant association between MRA treatment and reduced all-cause mortality in PEF patients (RD, -0.00 ; 95\% CI, -0.01 to $0.01 ; P=0.71 ; \mathrm{I}^{2}=0 \%$; Figure $2 \mathrm{~A}$, and RR, $0.90 ; 95 \%$ CI, 0.78 to $1.04 ; P=0.17 ; \mathrm{I}^{2}=0 \%$; Additional file 4: Figure S1A). Treatment with MRAs did not significantly reduce the incidence of all-cause mortality in the nine HF-PEF or in the five MI-PEF trials $(P=0.88$ and $P=0.60$, respectively). When analyzed by drug type, none of the individual therapies improved outcomes compared with the control group (spironolactone, $P=0.29$; canrenoate, $P=0.18$; eplerenone, $P=0.97$; Additional file 4: Figure S1B). None of the individual studies significantly influenced the pooled all-cause mortality estimate in the leave-one-out sensitivity analysis (Additional file 4: Figure S1C); publication bias was not observed $(P=0.31$, Additional file 4: Figure S1D).

Four studies [14,15,31,32] reported the outcomes of hospitalization due to HF involving 4,551 participants and 472 events. Overall, MRA treatment was associated with a reduced risk of hospitalization due to HF (RR, 0.83 ; $95 \% \mathrm{CI}, 0.70$ to $0.98 ; P=0.03 ; \mathrm{I}^{2}=0 \%$; Figure $2 \mathrm{~B}$, and RD, $-0.01 ; 95 \% \mathrm{CI},-0.03$ to $0.00 ; P=0.05 ; \mathrm{I}^{2}=0 \%$; Additional file 4: Figure S2A); a weight of $96.2 \%$ came from the TOPCAT trial. None of the individual studies influenced the pooled estimate of hospitalization due to
HF (Additional file 4: Figure S2B); publication bias was not observed ( $P=0.74$; Additional file 4: Figure S2C).

Four studies reported the composite outcomes for deaths due to cardiovascular causes, aborted cardiac arrest, or hospitalization due to HF. Overall, MRA treatment did not significantly reduce the incidence of the composite outcome (RR, $0.89 ; 95 \% \mathrm{CI}, 0.79$ to $1.01 ; P=0.07 ; \mathrm{I}^{2}=0 \%$, Additional file 4: Figure S3A). When analyzed according to PEF subtype, a significant benefit was not observed for either HF-PEF $(P=0.18)$ or MI-PEF $(P=0.14)$ patients. Given the marked regional variation in event rates observed in the TOPCAT trial, we performed a separate pooled analysis, excluding patients randomized into TOPCAT trial from Russia and the republic of Georgia. The results showed that MRA treatment significantly reduced the incidence of composite outcome of death from cardiovascular causes, aborted cardiac arrests, or hospitalizations due to HF in PEF patients (RR, 0.85; 95\% CI, 0.74 to 0.96; $P=0.01 ; \mathrm{I}^{2}=0 \%$; Additional file 4: Figure S3B).

\section{Effect of MRAs on echo indexes of diastolic function}

Overall, E/e', reported in 460 patients enrolled in four RCTs, was significantly improved following MRA treatment (WMD, -1.82 ; 95\% CI, -2.23 to $-1.42 ; \mathrm{I}^{2}=0 \%$; Figure $3 \mathrm{~A}$ ), without evidence of publication bias $(P=0.36)$. None of the individual studies significantly influenced the pooled estimate for E/e' (Additional file 4: Figure S4A). The E/A ratio was the most common diastolic function variable, reported in 1,535 patients enrolled in 10 RCTs. Using data from three trials involving MI-PEF patients, MRA treatment significantly improved the E/A ratio (WMD, 0.12; 95\% CI, 0.10 to 0.14; Figure 3B). However, the effect estimates for HF-PEF patients did not show improved E/A ratios $(P=0.97$; Figure $3 \mathrm{~B})$. When analyzed by drug type, canrenoate treatment was associated with significant improvement in the E/A ratio (WMD, 0.13; 95\% CI, 0.07 to 0.20; $\mathrm{I}^{2}=29.8 \%$; Additional file 4: Figure S4B). Treatment duration did not influence the pooled estimate (Additional file 4: Figure S4C).

For deceleration time, significance was attained among both HF-PEF (WMD, -14.35 ; 95\% CI, -28.65 to 0.06; $\mathrm{I}^{2}=75.8 \%$; Figure 3C) and MI-PEF (WMD, 6.70; 95\% CI, 3.45 to $9.95 ; \mathrm{I}^{2}=0 \%$; Figure $3 \mathrm{C}$ ) patients. Among the independent trials, overall estimates did not reach significance for IVRT ( $P=0.66$; Figure 3D).

\section{Effect of MRAs on indexes of cardiac structure and function}

With all trials included in the meta-analysis, improvement in LVEF (WMD, 2.22; 95\% CI, 1.35 to $3.10 ; \mathrm{I}^{2}=0 \%$; Figure 4A) and left ventricular end diastolic diameter (LVEDD) (SMD, $-0.21 ; 95 \% \mathrm{CI}, 0.32$ to $-0.11 ; \mathrm{I}^{2}=0 \%$; Figure 4B) was apparent. No publication bias was evident $(P=0.94$ and $P=0.61$, respectively; Additional file 4: 
Table 1 Characteristics of included studies

\begin{tabular}{|c|c|c|c|c|c|c|}
\hline Study (year) [Ref.] & Country & Patients & Inclusion criteria & Intervention: dose $(\mathrm{mg} / \mathrm{d})$ & $\begin{array}{l}\text { Number randomized } \\
\text { (withdrawals or dropouts) }\end{array}$ & $\begin{array}{l}\text { Follow-up } \\
\text { (months) }\end{array}$ \\
\hline \multirow[t]{2}{*}{ Grand I (2002) [26] } & \multirow[t]{2}{*}{ USA } & \multirow[t]{2}{*}{ HF-PEF and HBP } & \multirow{2}{*}{$\begin{array}{l}\text { LVEF > 50\%; BP > 140/90 mmHg; LV } \\
\text { diastolic dysfunction; normal renal function }\end{array}$} & Canrenone:50 & $17(\mathrm{NR})$ & \multirow[t]{2}{*}{6} \\
\hline & & & & ACEI & $17(\mathrm{NR})$ & \\
\hline \multirow[t]{2}{*}{ Mottram (2004) [27] } & \multirow[t]{2}{*}{ Australia } & \multirow[t]{2}{*}{ HF-PEF and HBP } & \multirow[t]{2}{*}{ LVEF > 50\%; NYHA II; E/A <1 } & Spironolactone:25 & 15 (NR) & \multirow[t]{2}{*}{6} \\
\hline & & & & Placebo & 15 (NR) & \\
\hline \multirow[t]{2}{*}{ Roongsritong (2005) [28] } & \multirow[t]{2}{*}{ USA } & \multirow[t]{2}{*}{ Elderly with HF-PEF } & \multirow{2}{*}{$\begin{array}{l}\text { LVEF >45\%; Age:60-85 years; mild diastolic } \\
\text { dysfunction }\end{array}$} & Spironolactone:25 & $15(1)$ & \multirow[t]{2}{*}{4} \\
\hline & & & & Placebo & $15(1)$ & \\
\hline \multirow[t]{2}{*}{ Orea-Tejeda (2007) [29] } & \multirow[t]{2}{*}{ Mexico } & \multirow[t]{2}{*}{ HF-PEF } & \multirow[t]{2}{*}{ LVEF $>40 \%$; shortening fraction $=28 \%$} & Spironolactone:25-50 & 14 (NR) & \multirow[t]{2}{*}{13.79} \\
\hline & & & & Standard therapy & 14 (NR) & \\
\hline \multirow[t]{2}{*}{ Mak (2009) [30] } & \multirow[t]{2}{*}{ Ireland } & \multirow[t]{2}{*}{ HF-PEF } & \multirow{2}{*}{$\begin{array}{l}\text { LVEF > } 45 \% \text {; NYHA IV; BNP } \geq 100 \text { pg/mL; } \\
\text { diastolic dysfunction }\end{array}$} & Eplerenone:25 & $24(0)$ & \multirow[t]{2}{*}{12} \\
\hline & & & & Standard therapy & $20(2)$ & \\
\hline \multirow[t]{2}{*}{ RAAM-PEF trial (2011) [31] } & \multirow[t]{2}{*}{ USA } & \multirow[t]{2}{*}{ HF-PEF } & \multirow[t]{2}{*}{ LVEF > 50\%; NYHA II or III; BNP $\geq 100$ pg/mL } & Eplerenone:25 (titrated to 50) & $23(0)$ & \multirow[t]{2}{*}{7} \\
\hline & & & & Placebo & $23(2)$ & \\
\hline \multirow[t]{2}{*}{ Aldo-DHF trial (2013) [16] } & Germany and Austria & HFPEF & LVEF > 50\%; NYHA II or III; Diastolic & Spironolactone:25 & $213(9)$ & 11.6 \\
\hline & & & aystunction grade $=$ & Placebo & $209(13)$ & \\
\hline Kurrelmeyer (2014) [32] & USA & Elderly women & LVEF >50\%; NYHA II or III; E/e' > 15; BNP & Spironolactone:25 & $24(0)$ & 6 \\
\hline & & & & Placebo & $24(0)$ & \\
\hline TOPCAT trial (2014) [14] & Americas, Russia, & HF-PEF & LVEF > 45\%; control blood pressure; BNP & Spironolactone:15-45 & $1722(160)$ & 39.6 \\
\hline & & & diastolic dysfunction (grade $\geq \mathrm{I}$ ) & Placebo & $1723(151)$ & \\
\hline DiPasquale (2005) [18] & Italy & MI-PEF & LVEF > $40 \%$; ST > $1 \mathrm{~mm}$ in the peripheral & Canrenoate:25 & $341(33)$ & 6 \\
\hline & & & leads and/or > 2 mm in precoralal leads & placebo & $346(30)$ & \\
\hline Kayrak (2010) [19] & Turkey & MI-PEF & LVEF $>40 \%$; successfully revascularized & Spironolactone:25 & $71(16)$ & 6 \\
\hline & & & & Standard therapy & $71(16)$ & \\
\hline Kampourides (2012) [17] & Greece & MI-PEF & LVEF >40\%; AMI 1 day to 7 days & Eplerenone:25 & $210(9)$ & 30 \\
\hline & & & & Standard therapy & $140(38)$ & \\
\hline Vatankulu (2013) [5] & Turkey & MI-PEF & LVEF $>40 \%$; successfully revascularized & Spironolactone:25 & 54 (NR) & 6 \\
\hline & & & patlents WItn AIVII & Standard therapy & $56(N R)$ & \\
\hline REMINDER trial (2014) [15] & European countries & MI-PEF & LVEF $>40 \%$; successfully revascularized & Eplerenone: 25 (titrated to 50) & $506(82)$ & 10.5 \\
\hline & & & pacientis witui Mivi & Placebo & $506(79)$ & \\
\hline
\end{tabular}

ACEl, Angiotensin-converting enzyme inhibitor; AMI, Myocardial infarction; BNP, B-type natriuretic peptide; E/A ratio, the ratio of early to late diastolic transmitral flow; E/e', an echocardiographic estimate of filling pressure for assessment of diastolic function; HBP, High blood pressure; HF-PEF, Heart failure with preserved systolic function; LVEF, Left ventricular ejection fraction; MI-PEF, Myocardial infarction with preserved systolic function; NR, Not reported; NT-proBNP, Amino terminal pro-B-type natriuretic peptide; NYHA, New York Heart Association functional class. 
A

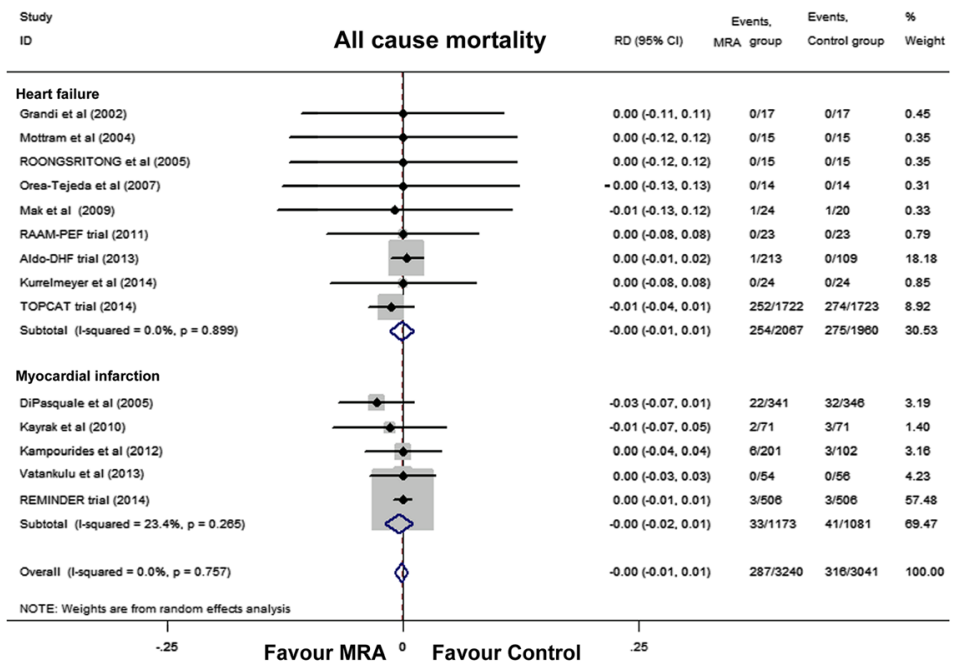

B

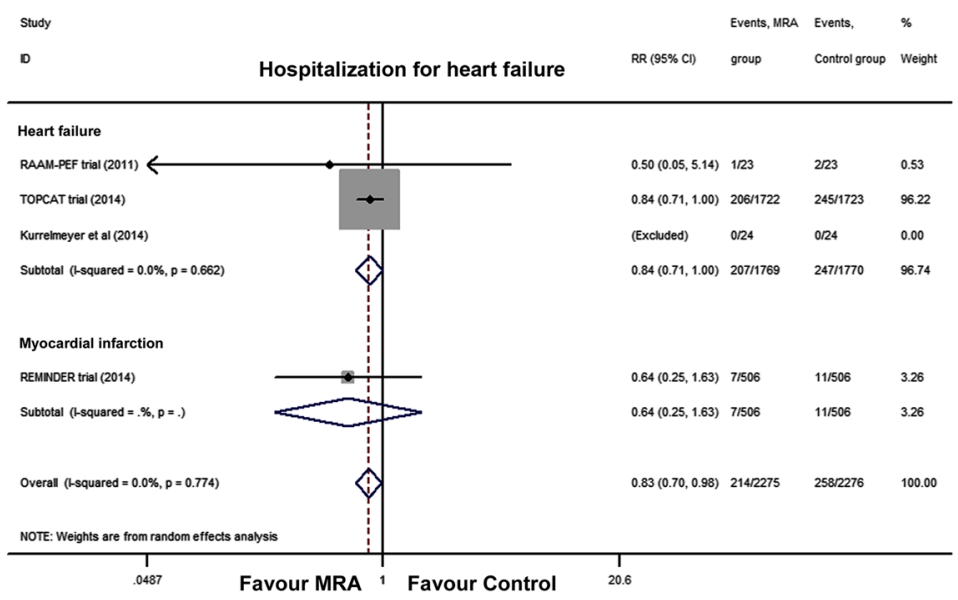

Figure 2 Pooled analyses of all-cause mortality and hospitalization for heart failure in the mineralocorticoid receptor antagonist group versus controls. (A) All-cause hospitalization; (B) Hospitalization due to heart failure. Cl, Confidence interval; MRA, Mineralocorticoid receptor antagonist; RD, Absolute risk reduction; RR, Relative risk.

Figure S5A and S5B, respectively). Subgroup analyses, according to PEF subtype, found that the improvement in LVEF remained significant in both the HF-PEF and MI-PEF groups (Figure 4B). Subgroup analysis by treatment duration found that the MRA benefit on LVEDD was significant over 6 months, but not over 12 months (Additional file 4: Figure S5C).

For LAVI, the overall effect estimates did not demonstrate any significant benefit of MRA treatment (WMD, $-0.11 ; 95 \% \mathrm{CI},-0.27$ to $0.06 ; \mathrm{I}^{2}=0 \% ; P=0.26$; Figure $4 \mathrm{C}$ ). Similarly, MRA treatment was not associated with significant LVMI improvement (WMD, -0.12; $95 \% \mathrm{CI},-0.28$ to $0.05 ; \mathrm{I}^{2}=0 \% ; P=0.22$; Figure $\left.4 \mathrm{D}\right)$. Further subgroup analyses failed to demonstrate significance for LAVI and LVMI (Figure 4D).
Effect of MRAs on functional capacity and serum indicators The pooled analyses of QoL and 6-min walk distance are shown in Figure 5A and B, respectively. When the QoL was measured using the Kansas City Cardiomyopathy Questionnaire clinical summary score (KCCQ CSS), MRA treatment was associated with a significant improvement in QoL in HF-PEF patients (WMD, -5.16 ; 95\% CI, -8.03 to $-2.30 ; \mathrm{I}^{2}=0 \% ; P<0.0001$; Figure $\left.5 \mathrm{~A}\right)$. However, the Minnesota Living with Heart Failure questionnaire QoL measurements did not improve significantly. In the three HF-PEF trials that reported 6-min walk distance, a nonsignificant change was seen (WMD, -7.97 ; 95\% CI, -16.51 to $0.57 ; \mathrm{I}^{2}=0 \% ; P=0.07$; Figure $5 \mathrm{~B}$ ).

Pooling the results of all qualified trials found a significant serum PIIINP level reduction (WMD, -1.50; 


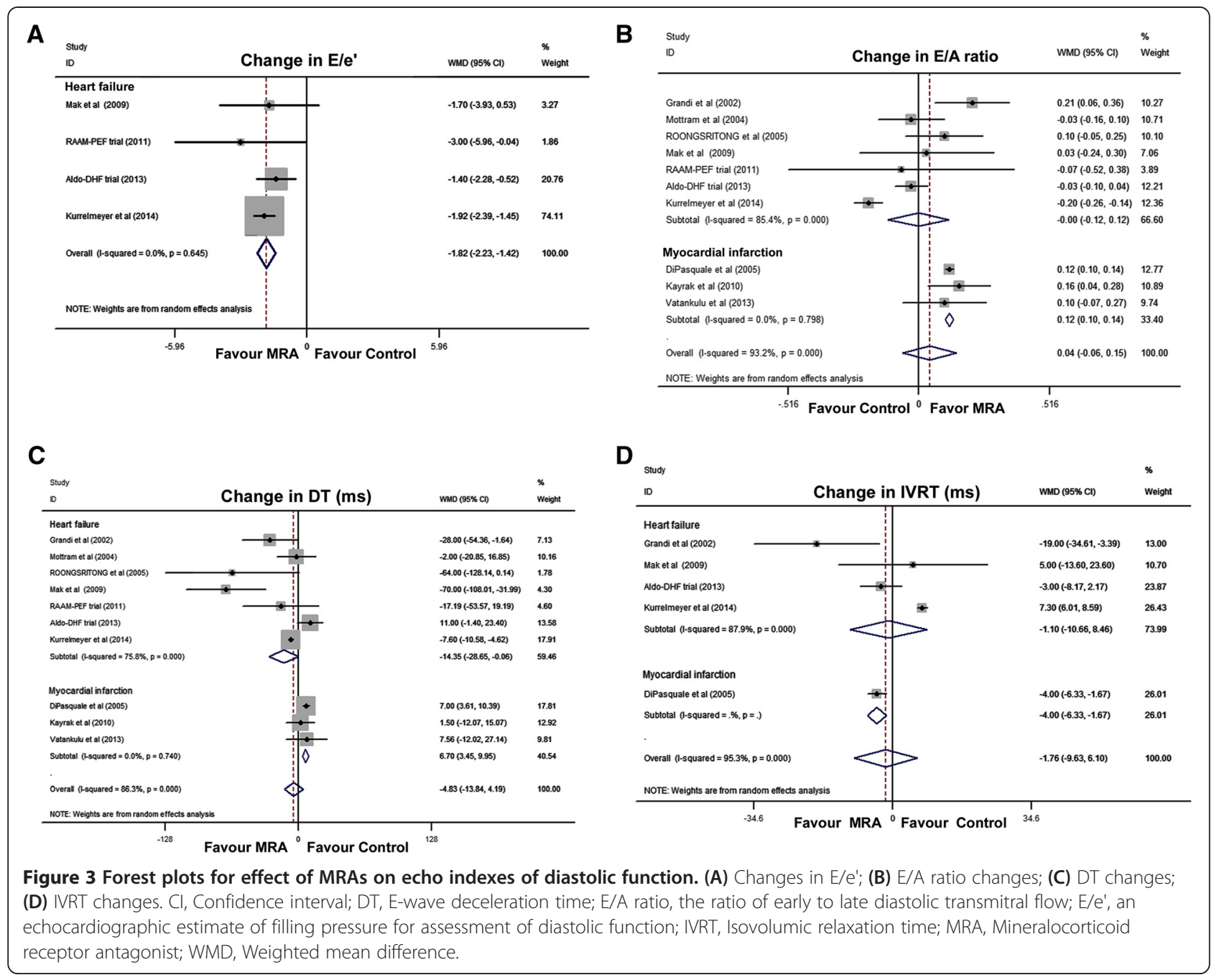

95\% CI, -1.72 to -1.29 ; $\mathrm{I}^{2}=0 \%$; Figure $5 \mathrm{C}$ ), with significant publication bias $(P=0.01)$. As for B-type natriuretic peptide (or amino terminal-proB-type natriuretic peptide), the pooled analysis did not show a significant reduction in the MRA group (WMD, -4.83 ; 95\% CI, -46.12 to $36.45 ; \mathrm{I}^{2}=52.8 \% ; P=0.82$; Figure $\left.5 \mathrm{D}\right)$.

\section{Safety and adverse events}

Hyperkalemia was reported in all but one trial [28]. Over a mean follow-up period of 12.19 months, the MRA group showed a higher rate of hyperkalemia $(>5.5 \mathrm{mmol} / \mathrm{L})$ with $12.15 \%$ of the MRA groups and $6.16 \%$ of the control groups reporting hyperkalemia $(P<0.001)$. The TOPCAT trial, which had the highest reported rates of hyperkalemia, involved spironolactone treatment (15 to $45 \mathrm{mg}$ ). Renal failure, using definitions from within each trial, occurred in $1.91 \%$ of MRA patients and in $0.37 \%$ of control patients. Gynecomastia was reported in seven studies $[5,14,16,19,26,29,31]$ involving MRA (2.81\%) and control (0.30\%) patients.

\section{Discussion}

In this meta-analysis of RCTs involving 6,248 patients, the effects of MRAs on patients with either MI-PEF or HF-PEF were evaluated. MRA treatment reduced the risk of hospitalization due to $\mathrm{HF}$, improved QoL, reduced the E/e' or E/A ratio, increased LVEF, and reduced LVEDD and PIIINP levels in PEF patients. However, significant allcause mortality benefits were not seen.

As patients with HF-PEF are usually older than HF-REF patients, hospitalization due to HF is increasing and represents a major burden in these patients $[1,33]$, and emphasizes the growing need for effective, evidence-based therapies. However, previous pharmacological interventions, such as angiotensin-converting enzyme inhibitors [34], angiotensin receptor blockers [35], and beta-blockers [36], have failed to show a significant reduction in hospitalizations due to HF. This meta-analysis provides important insights into the potential efficacy of MRA treatment for reducing the rate of hospitalizations due to HF in PEF patients, without increasing mortality. Reducing 


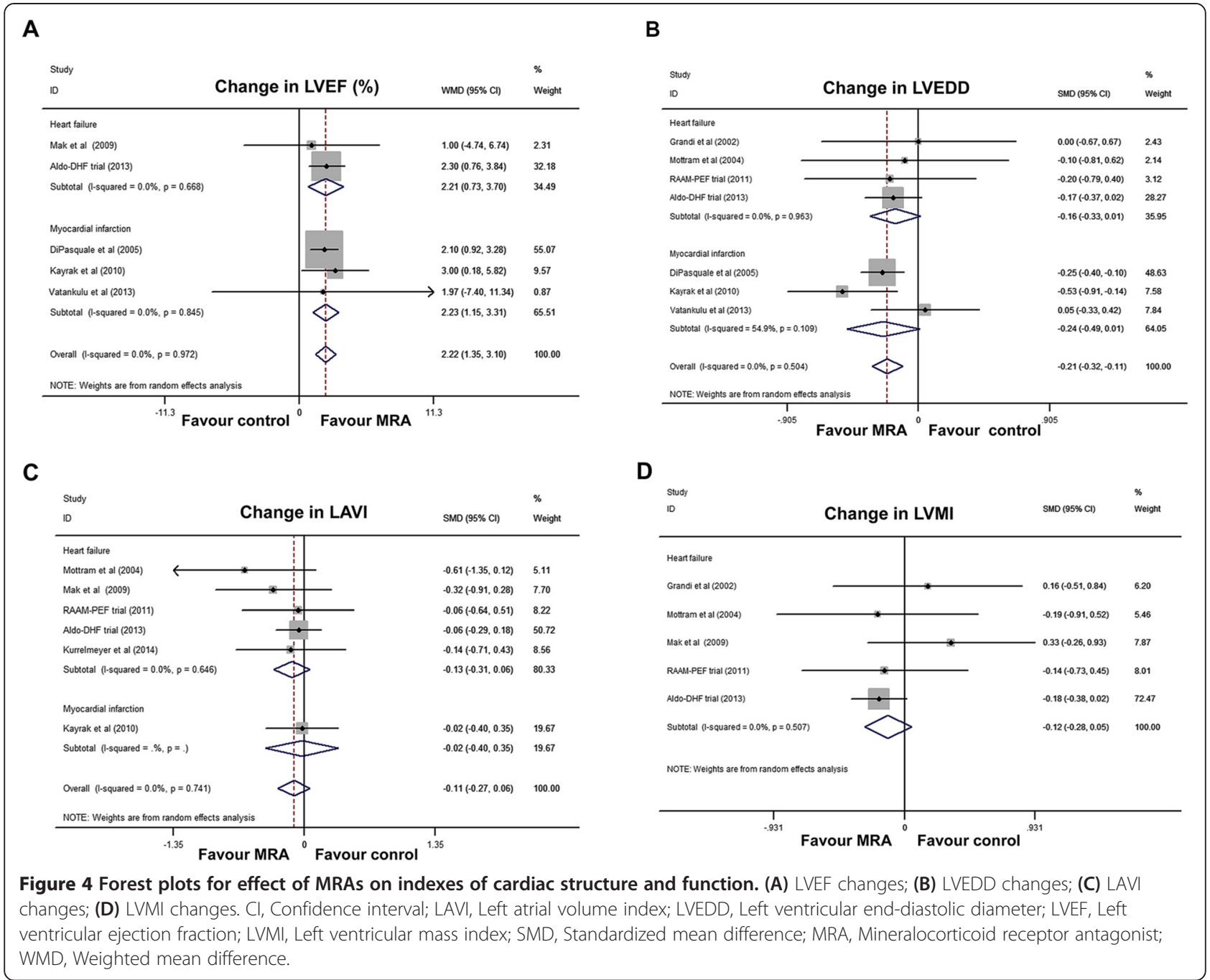

hospitalizations due to HF may help lower hospitalization costs and improve patient QoL. Additionally, significant MRA treatment benefits on composite outcome of death from cardiovascular causes, aborted cardiac arrests, or hospitalizations were observed after excluding patients recruited from Russia and the Republic of Georgia into the TOPCAT trial. HF-PEF patients from these jurisdictions, in that trial [14], had extremely low placebo event rates, incompatible with those in prior HF-PEF studies [35,37]. The separate meta-analysis, excluding this population, might provide a more realistic insight into the effectiveness of MRAs in HF-PEF patients. Furthermore, we demonstrated that MRA treatment was associated with a significant improvement in QoL, measured by the KCCQ CCS. The KCCQ CCS has been reported to be a valid and reliable measure of health status and QoL in HF-PEF patients [38]. Since the HF-PEF patients were elderly and typically demonstrated multiple comorbidities that might affect their mobility, it is not surprising that MRA treatment improved KCCQ CCS scores in these patients, but not exercise tolerance [39]. Therefore, MRA treatment could be an option in PEF patients to improve their QoL.

Another encouraging finding from this meta-analysis was that MRAs improved both diastolic and systolic functions in PEF patients. Left ventricular diastolic dysfunction is the major underlying cardiac pathophysiology of PEF patients, and worse diastolic dysfunction has been associated with an increased risk of mortality [40]. However, earlier pharmacotherapy did not achieve a significant improvement in diastolic function in HF-PEF patients $[7,41,42]$. The present meta-analysis supports the potential clinical value of MRAs for improving diastolic function in PEF patients. We also found that MRA treatment significantly reduced the E/e' in HF-PEF patients and the E/A ratio in MI-PEF patients. Interestingly, MRAs were not associated with a significant reduction in the E/A ratio in HF-PEF patients. This may be because this ratio is rather complicated and cannot provide unequivocal evidence of diastolic dysfunction in HF-PEF [43], whereas E/e' was demonstrated to be the 


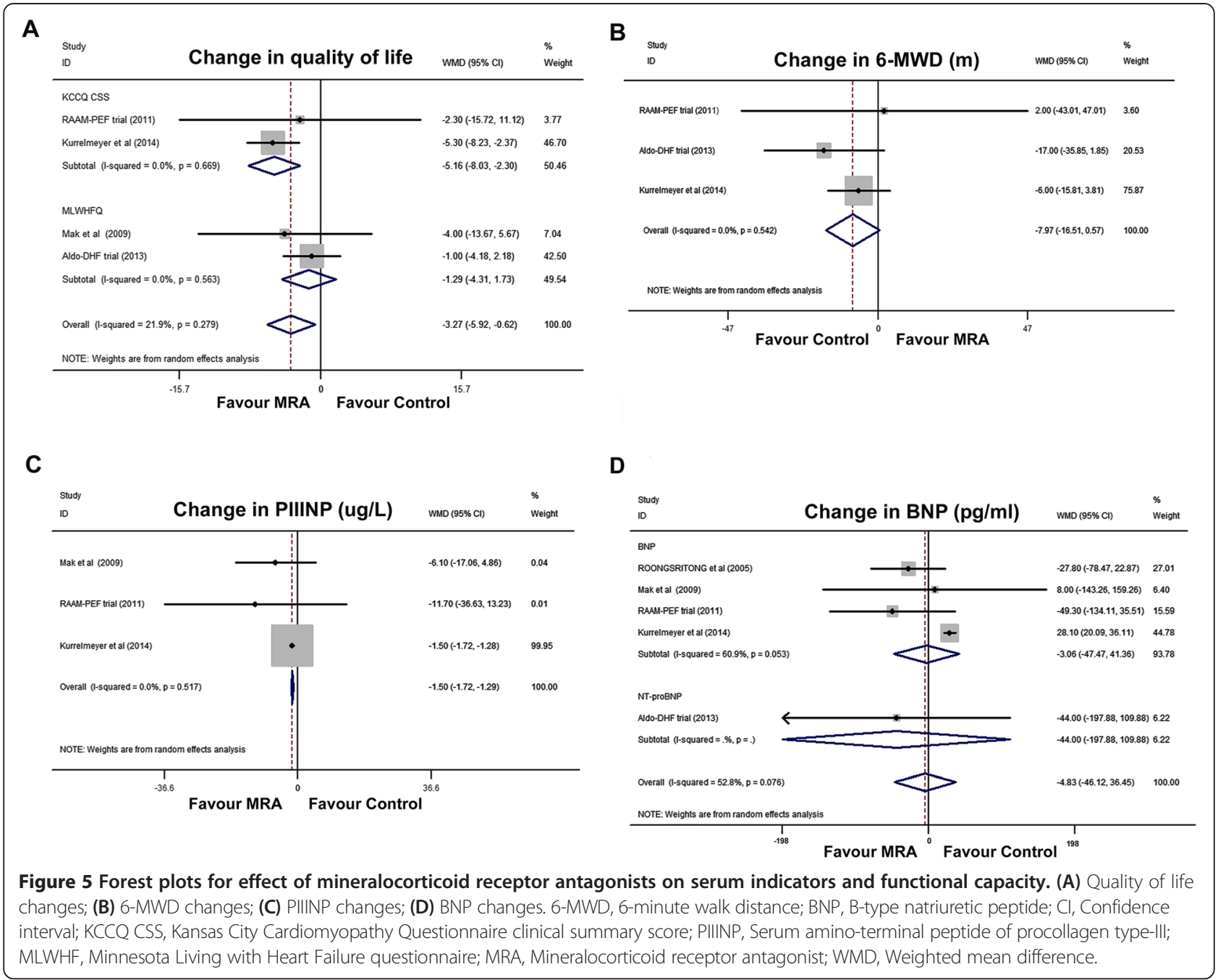

most accurate, non-invasive index of diastolic function in HF-PEF [43]. Our meta-analysis also found that MRA administration led to increased LVEF in PEF patients. A previous meta-analysis demonstrated that MRAs improved systolic function in HF-REF [44]. Thus, the present meta-analysis shows that the beneficial effect of MRAs on systolic function also extends to PEF patients.

Although the favorable impact of MRAs on cardiac remodeling is well known in patients with HF and reduced LVEF [44], the effect of MRAs in patients with preserved systolic function remained uncertain. This meta-analysis demonstrated that MRA administration could reverse cardiac remodeling in patients with preserved systolic function through a reduction of LVEDD and PIIINP levels. A subgroup analysis of LVEDD, based on treatment duration, found that the reduction became insignificant as the duration increased. This finding is consistent with a previous meta-analysis focusing on the effect of MRAs on cardiac structure in patients with left ventricular dysfunction [44]. As PIIINP level has been proposed as an indicator of cardiac remodeling and poor clinical prognosis [45], a reduction in serum PIIINP level might reflect the beneficial effects of MRAs on cardiac remodeling.

The benefits of MRAs on PEF patients are mainly attributed to the improvement of endothelial function and cardiac remodeling, as well as the decrease of myocardial fibrosis. Experimental evidence indicates that aldosteroneinduced mineralocorticoid receptor activation provides an important unifying mechanism for many of the pathologic alterations of HF-PEF and MI-PEF [46,47]. The MRAs, through direct inhibition of aldosterone, were demonstrated to reduce myocardial fibrosis, improve vascular compliance and endothelial function, decrease inflammation and oxidative stress, and reduce the release of norepinephrine [48]. These changes likely account for the diastolic function improvements seen on echocardiography and the reduction in collagen markers such as serum PIIINP level. MRA treatment was also associated with an increased risk of hyperkalemia and elevated serum creatinine levels. Our findings underscore the importance 
of monitoring electrolyte disorders and serum markers of kidney function during MRA treatment in clinical practice.

Several issues should be considered in the interpretation of our results. First, this meta-analysis was limited by the discrepancies in PEF diagnostic criteria employed in the clinical trials. The diagnosis of HF-PEF is still challenging because various criteria have been proposed to define patients with "diastolic HF" [49]. The included RCTs had differing ejection fraction cut-off criteria (range, 40 to $50 \%$ ) and challenges in diagnostic criteria for PEF, and may have resulted in a heterogeneous population. Nevertheless, patients with an ejection fraction of 40 to $50 \%$, defined as borderline and intermediate PEF in the American College of Cardiology Foundation/American Heart Association guidelines [49], were characteristically and prognostically similar to those with an ejection fraction $\geq 50 \%$ [50]. Therefore, our meta-analysis does suggest a potential MRA treatment benefit for PEF patients. Second, some publication bias might exist in this meta-analysis, as we only included articles published in English. However, our statistical tests reported a low probability of publication bias in the pooling analysis. Third, different follow-up durations in the included trials might have produced heterogeneity, which limited the interpretation of pooled effect estimates. Finally, as the reported totals for all-cause mortality and hospitalizations due to HF were low, the assessment of the effect on clinical outcomes in PEF patients was of limited power. Despite the majority of evidence regarding clinical outcomes coming from the recently reported TOPCAT trial [14], the findings of previous trials appear consistent.

\section{Conclusions}

This meta-analysis of RCTs in PEF patients demonstrated that MRA treatment may exert beneficial effects, including reduced hospitalizations due to HF, improved QoL and diastolic function, and cardiac remodeling reversal, without an effect on all-cause mortality. The significant increase in hyperkalemia and serum creatinine level associated with MRA treatment underscores the need for careful monitoring of electrolyte disorders and serum markers of kidney function in clinical practice. Further large-scale RCTs are needed to confirm the clinical indications for this medication class.

\section{Additional files}

Additional file 1: PRISMA checklist.

Additional file 2: Search Strategies.

Additional file 3: Table S1. Quality Assessment of included RCTs in this meta-analysis. Table S2. Characteristics of patients in the included studies. Table S3. Characteristics of patients in the included studies.
Additional file 4: Figure S1. Effect of MRAs on all-cause mortality. (A) Pooled effect estimate RR on all-cause mortality; (B) Subgroup analysis by drug type; (C) Leave-one-out analysis; (D) Funnel plots for all-cause mortality. Cl, Confidence interval; MRA, Mineralocorticoid receptor antagonist; RR, Relative risk; SE, Standard error. Figure S2. Effect of MRAs on hospitalization for heart failure. (A) Pooled effect estimate RD on hospitalization for heart failure; (B) Leave-one-out analysis; (C)Funnel plots for hospitalization for heart failure. $\mathrm{Cl}$, Confidence interval; MRA, Mineralocorticoid receptor antagonist; RD, Absolute risk reduction; SE, Standard error. Figure S3. Effect of MRAs on composite outcomes for deaths due to cardiovascular causes, aborted cardiac arrest, or hospitalization due to heart failure. (A) Pooled effect estimate RR on composite outcomes; (B) Pooled analysis excluding patients randomized into TOPCAT trial from Russia and the Republic of Georgia on composite outcomes.Cl, Confidence interval; MRA, Mineralocorticoid receptor antagonist; RR, Relative risk; SE, Standard error. Figure S4. Funnel plots and subgroup analyses of echo indexes of diastolic function. (A) Leaveone-out analysis for E/e'; (B) Subgroup analyses by drug type; (C) Subgroup analyses by treatment duration. $\mathrm{Cl}$, Confidence interval; E/A ratio, the ratio of early to late diastolic transmitral flow; E/e', an echocardiographic estimate of filling pressure for assessment of diastolic function; MRA, Mineralocorticoid receptor antagonist; WMD, Weighted mean difference. Figure S5. Subgroup analyses of indexes of cardiac structure and function. (A) Funnel plots for LVEF; (B) Subgroup analyses by LVEDD; (C) Subgroup analyses by treatment duration. LVEDD, Left ventricular end-diastolic diameter; LVEF, Left ventricular ejection fraction; LVMI, Left ventricular mass index; SMD, Standardized mean difference; MRA, Mineralocorticoid receptor antagonist; WMD, Weighted mean difference.

\section{Abbreviations}

Cls: Confidence intervals; E/A: Ratio of early to late diastolic transmitral flow; E/e': Estimate of filling pressure used to assess diastolic function; HF: Heart failure; HF-PEF: HF with preserved ejection fraction; HF-REF: HF patients with a reduced ejection fraction; IVRT: Isovolumic relaxation time; KCCQ CSS: Kansas City Cardiomyopathy Questionnaire clinical summary score; LAVI: Left atrial volume index; LVEDD: Left ventricular end diastolic diameter; LVEFs: Left ventricular ejection fractions; LVMI: Left ventricular mass index; MI: Myocardial infarction; MI-PEF: PEF after MI; MRAs: Mineralocorticoid receptor antagonists; PEF: Preserved ejection fraction; PIIINP: Peptide of procollagen type-III; QoL: Quality of life; RCTs: Randomized controlled trials; RD: Risk reduction; RR: Relative risk; SMD: Standardized mean difference; TOPCAT: Treatment of Preserved Cardiac Function Heart Failure with an Aldosterone Antagonist; WMD: Weighted mean difference.

\section{Competing interests}

The authors declare that they have no competing interests.

\section{Authors' contributions}

YC and JB conceived and designed this meta-analysis. YC, HW, and YL performed the literature search and data extraction. YC, HW, XH, and JB conducted statistical analyses and data interpretations. YC and HW drafted the manuscript. YL and JB provided supervision. All authors critically revised the manuscript. All authors approved the final version of the manuscript submitted for publication and are guarantors of the study.

\section{Acknowledgements}

This study was supported by grants to JPB from the National Basic Research Program of China (973 Program) (No. 2013CB733804), the National Natural Science Foundation of China (No. 81227801 and No. 81271640), and the Team Program of the Natural Science Foundation of Guangdong Province, China (S2011030003134).

Received: 24 September 2014 Accepted: 17 December 2014 Published online: 19 January 2015

\section{References}

1. Owan TE, Hodge DO, Herges RM, Jacobsen SJ, Roger VL, Redfield MM. Trends in prevalence and outcome of heart failure with preserved ejection fraction. New Engl J Med. 2006;355:251-9. 
2. Redfield MM, Jacobsen SJ, Burnett Jr JC, Mahoney DW, Bailey KR, Rodeheffer RJ. Burden of systolic and diastolic ventricular dysfunction in the community: appreciating the scope of the heart failure epidemic. JAMA. 2003;289:194-202.

3. Lam CS, Donal E, Kraigher-Krainer E, Vasan RS. Epidemiology and clinical course of heart failure with preserved ejection fraction. Eur J Heart Fail. 2011;13:18-28.

4. Hoekstra T, Lesman-Leegte I, van Veldhuisen DJ, Sanderman R, Jaarsma T. Quality of life is impaired similarly in heart failure patients with preserved and reduced ejection fraction. Eur J Heart Fail. 2011;13:1013-8.

5. Vatankulu MA, Bacaksiz A, Sonmez O, Alihanoglu Y, Koc F, Demir K, et al. Does spironolactone have a dose-dependent effect on left ventricular remodeling in patients with preserved left ventricular function after an acute myocardial infarction? Cardiovasc Therapeut. 2013;31:224-9.

6. Yancy CW, Jessup M, Bozkurt B, Butler J, Casey Jr DE, Drazner MH, et al. ACCF/AHA guideline for the management of heart failure: executive summary: a report of the American College of Cardiology Foundation/ American Heart Association Task Force on practice guidelines. Circulation. 2013;2013:1810-52.

7. Holland DJ, Kumbhani DJ, Ahmed SH, Marwick TH. Effects of treatment on exercise tolerance, cardiac function, and mortality in heart failure with preserved ejection fraction. A meta-analysis. J Am Coll Cardiol. 2011;57:1676-86

8. Borlaug BA, Paulus WJ. Heart failure with preserved ejection fraction: pathophysiology, diagnosis, and treatment. Eur Heart J. 2011;32:670-9.

9. Edelmann F, Tomaschitz A, Wachter R, Gelbrich G, Knoke M, Dungen HD, et al. Serum aldosterone and its relationship to left ventricular structure and geometry in patients with preserved left ventricular ejection fraction. Eur Heart J. 2012;33:203-12.

10. Delyani JA. Mineralocorticoid receptor antagonists: the evolution of utility and pharmacology. Kidney Int. 2000;57:1408-11.

11. Pitt B, Zannad F, Remme WJ, Cody R, Castaigne A, Perez A, et al. The effect of spironolactone on morbidity and mortality in patients with severe heart failure. Randomized Aldactone Evaluation Study Investigators. New Engl J Med. 1999;341:709-17.

12. Zannad F, McMurray JJ, Krum H, van Veldhuisen DJ, Swedberg K, Shi H, et al. Eplerenone in patients with systolic heart failure and mild symptoms. New Engl J Med. 2011;364:11-21.

13. Pitt B, Remme W, Zannad F, Neaton J, Martinez F, Roniker B, et al. Eplerenone, a selective aldosterone blocker, in patients with left ventricular dysfunction after myocardial infarction. New Engl J Med. 2003;348:1309-21.

14. Pitt B, Pfeffer MA, Assmann SF, Boineau R, Anand IS, Claggett B, et al. Spironolactone for heart failure with preserved ejection fraction. New Engl J Med. 2014;370:1383-92.

15. Montalescot G, Pitt B, Lopez de Sa E, Hamm CW, Flather M, Verheugt F, et al. Early eplerenone treatment in patients with acute ST-elevation myocardial infarction without heart failure: The Randomized Double-Blind Reminder Study. Eur Heart J. 2014;35:2295-302.

16. Edelmann F, Wachter R, Schmidt AG, Kraigher-Krainer E, Colantonio C, Kamke W, et al. Effect of spironolactone on diastolic function and exercise capacity in patients with heart failure with preserved ejection fraction: the Aldo-DHF randomized controlled trial. JAMA. 2013;309:781-91.

17. Kampourides $N$, Tziakas D, Chalikias G, Papazoglou D, Maltezos $E_{t}$ Symeonides D, et al. Usefulness of matrix metalloproteinase-9 plasma levels to identify patients with preserved left ventricular systolic function after acute myocardial infarction who could benefit from eplerenone. Am J Cardiol. 2012;110:1085-91.

18. Di Pasquale P, Cannizzaro S, Scalzo S, Parrinello G, Fasullo S, Giambanco F, et al. Effects of canrenoate plus angiotensin-converting enzyme inhibitors versus angiotensin-converting enzyme inhibitors alone on systolic and diastolic function in patients with acute anterior myocardial infarction. Am Heart J. 2005;150:919.

19. Kayrak M, Bacaksiz A, Vatankulu MA, Ayhan SS, Ari H, Kaya Z, et al. The effects of spironolactone on atrial remodeling in patients with preserved left ventricular function after an acute myocardial infarction: a randomized follow-up study. Coron Artery Dis. 2010;21:477-85.

20. Zile MR, Baicu CF, Gaasch WH. Diastolic heart failure-abnormalities in active relaxation and passive stiffness of the left ventricle. New Engl J Med. 2004:350:1953-9.
21. Moher D, Liberati A, Tetzlaff J, Altman DG, PRISMA Group. Preferred reporting items for systematic reviews and meta-analyses: the PRISMA statement. Int J Surg. 2010;8:336-41.

22. Jadad AR, Moore RA, Carroll D, Jenkinson C, Reynolds DJ, Gavaghan DJ, et al. Assessing the quality of reports of randomized clinical trials: is blinding necessary? Contr Clin Trials. 1996;17:1-12.

23. DerSimonian R, Kacker R. Random-effects model for meta-analysis of clinical trials: an update. Contemp Clin Trials. 2007;28:105-14.

24. Higgins JP, Thompson SG, Deeks JJ, Altman DG. Measuring inconsistency in meta-analyses. BMJ. 2003;327:557-60.

25. Egger M, Davey Smith G, Schneider M, Minder C. Bias in meta-analysis detected by a simple, graphical test. BMJ. 1997;315:629-34.

26. Grandi AM, Imperiale D, Santillo R, Barlocco E, Bertolini A, Guasti L, et al. Aldosterone antagonist improves diastolic function in essential hypertension. Hypertension. 2002;40:647-52.

27. Mottram PM, Haluska B, Leano R, Cowley D, Stowasser M, Marwick TH. Effect of aldosterone antagonism on myocardial dysfunction in hypertensive patients with diastolic heart failure. Circulation. 2004;110:558-65.

28. Roongsritong C, Sutthiwan P, Bradley J, Simoni J, Power S, Meyerrose GE. Spironolactone improves diastolic function in the elderly. Clin Cardiol. 2005;28:484-7.

29. Orea-Tejeda A, Colin-Ramirez E, Castillo-Martinez L, Asensio-Lafuente E, Corzo-Leon D, Gonzalez-Toledo R, et al. Aldosterone receptor antagonists induce favorable cardiac remodeling in diastolic heart failure patients. Rev Invest Clin. 2007;59:103-7.

30. Mak GJ, Ledwidge MT, Watson CJ, Phelan DM, Dawkins IR, Murphy NF, et al. Natural history of markers of collagen turnover in patients with early diastolic dysfunction and impact of eplerenone. J Am Coll Cardiol. 2009;54:1674-82

31. Deswal A, Richardson P, Bozkurt B, Mann DL. Results of the Randomized Aldosterone Antagonism in Heart Failure with Preserved Ejection Fraction trial (RAAM-PEF). J Card Fail. 2011;17:634-42.

32. Kurrelmeyer KM, Ashton Y, Xu J, Nagueh SF, Torre-Amione G, Deswal A. Effects of spironolactone treatment in elderly women with heart failure and preserved left ventricular ejection fraction. J Card Fail. 2014;20:560-8.

33. Steinberg BA, Zhao $X$, Heidenreich PA, Peterson ED, Bhatt DL, Cannon CP, et al. Get With the Guidelines Scientific Advisory Committee and Investigators. Trends in patients hospitalized with heart failure and preserved left ventricular ejection fraction: prevalence, therapies, and outcomes. Circulation. 2012;126:65-75.

34. Fu M, Zhou J, Sun A, Zhang S, Zhang C, Zou Y, et al. Efficacy of ACE inhibitors in chronic heart failure with preserved ejection fraction-a meta-analysis of 7 prospective clinical studies. Int J Cardiol. 2012;155:33-8.

35. Massie BM, Carson PE, McMurray JJ, Komajda M, McKelvie R, Zile MR, et al. Irbesartan in patients with heart failure and preserved ejection fraction. New Engl J Med. 2008;359:2456-67.

36. Liu F, Chen Y, Feng X, Teng Z, Yuan Y, Bin J. Effects of beta-blockers on heart failure with preserved ejection fraction: a meta-analysis. PLoS One. 2014;9:e90555.

37. Yusuf S, Pfeffer MA, Swedberg K, Granger CB, Held P, McMurray JJ, et al. CHARM Investigators and Committees. Effects of candesartan in patients with chronic heart failure and preserved left-ventricular ejection fraction: the CHARM-Preserved Trial. Lancet. 2003;362:777-81.

38. Joseph SM, Novak E, Arnold SV, Jones PG, Khattak H, Platts AE, et al. Comparable performance of the Kansas City Cardiomyopathy Questionnaire in patients with heart failure with preserved and reduced ejection fraction. Circ Heart Fail. 2013;6:1139-46.

39. Shah SJ, Gheorghiade M. Heart failure with preserved ejection fraction: treat now by treating comorbidities. JAMA. 2008;300:431-3.

40. Schillaci G, Pasqualini L, Verdecchia P, Vaudo G, Marchesi S, Porcellati C, et al. Prognostic significance of left ventricular diastolic dysfunction in essential hypertension. J Am Coll Cardiol. 2002;39:2005-11.

41. Solomon SD, Janardhanan R, Verma A, Bourgoun M, Daley WL, Purkayastha $D$, et al. Effect of angiotensin receptor blockade and antihypertensive drugs on diastolic function in patients with hypertension and diastolic dysfunction: a randomised trial. Lancet. 2007:369:2079-87.

42. Conraads VM, Metra M, Kamp O, De Keulenaer GW, Pieske B, Zamorano J, et al. Effects of the long-term administration of nebivolol on the clinical symptoms, exercise capacity, and left ventricular function of patients with diastolic dysfunction: results of the ELANDD study. Eur J Heart Fail. 2012;14:219-25. 
43. Kasner M, Westermann D, Steendijk P, Gaub R, Wilkenshoff U, Weitmann K, et al. Utility of Doppler echocardiography and tissue Doppler imaging in the estimation of diastolic function in heart failure with normal ejection fraction: a comparative Doppler-conductance catheterization study. Circulation. 2007;116:637-47.

44. Li X, Qi Y, Li Y, Zhang S, Guo S, Chu S, et al. Impact of mineralocorticoid receptor antagonists on changes in cardiac structure and function of left ventricular dysfunction: a meta-analysis of randomized controlled trials. Circ Heart Fail. 2013;6:156-65.

45. Zannad F, Alla F, Dousset B, Perez A, Pitt B. Limitation of excessive extracellular matrix turnover may contribute to survival benefit of spironolactone therapy in patients with congestive heart failure: insights from the randomized aldactone evaluation study (RALES). Rales Investigators. Circulation. 2000;102:2700-6.

46. Borlaug BA, Kass DA. Mechanisms of diastolic dysfunction in heart failure Trends Cardiovasc Med. 2006;16:273-9.

47. Sigurdsson A, Held P, Swedberg K. Short- and long-term neurohormonal activation following acute myocardial infarction. Am Heart J. 1993;126:1068-76.

48. Weber KT. Aldosterone in congestive heart failure. New Engl J Med. 2001;345:1689-97.

49. Yancy CW, Jessup M, Bozkurt B, Butler J, Casey Jr DE, Drazner MH, et al. 2013 ACCF/AHA guideline for the management of heart failure: a report of the American College of Cardiology Foundation/American Heart Association Task Force on Practice Guidelines. J Am Coll Cardiol. 2013;62:e147-239.

50. Fonarow GC, Stough WG, Abraham WT, Albert NM, Gheorghiade M, Greenberg BH, et al. Characteristics, treatments, and outcomes of patients with preserved systolic function hospitalized for heart failure: a report from the OPTIMIZE-HF Registry. J Am Coll Cardiol. 2007;50:768-77.

\section{Submit your next manuscript to BioMed Central and take full advantage of:}

- Convenient online submission

- Thorough peer review

- No space constraints or color figure charges

- Immediate publication on acceptance

- Inclusion in PubMed, CAS, Scopus and Google Scholar

- Research which is freely available for redistribution 